\title{
Improving the quality of life of care home residents with dementia: Cost effectiveness of an optimised intervention for residents with clinically significant agitation in dementia
}

Renee Romeo ${ }^{1 *}$, Darshan Zala ${ }^{2^{*}}$, Martin Knapp ${ }^{3}$, Martin Orrell ${ }^{4}$, Jane Fossey ${ }^{5}$, Clive Ballard 6

1Senior Lecturer in health economics, Health Service and Population Research Department, King's College London, institute of Psychiatry, Psychology and Neuroscience, London, UK ${ }^{2}$ Research Associate, Health Service and Population Research Department, King's College London, institute of Psychiatry, Psychology and Neuroscience, London, UK

${ }^{3}$ Professor of social policy, director of personal social services research unit, London School of Economics and Political Science, Houghton Street, London WC2A 2AE, UK

${ }^{4}$ Professor of ageing and mental health and Director - Institute of Mental Health, Faculty of Medicine \& Health Sciences, University of Nottingham, Innovation Park, Triumph Road, Nottingham, NG7 2TU, UK

${ }^{5}$ Associate Director of Psychological Services, Oxford Health NHS Foundation Trust, Fulbrook Centre, Oxford OX3 7JU, UK

${ }^{6}$ Professor of age related diseases, co-director of the biomedical research unit for dementia at the Institute of Psychiatry Wolfson Centre for Age Related Diseases, King's College London, Guy's Campus, London SE1 1UL, UK

*Joint first authors

Corresponding author: Dr Renee Romeo, DeCrespigny Park, Denmark Hill, SE 5 8AF, Tel: 0207848 5082, renee.romeo@kcl.ac.uk

Word count: 3876 


\section{Abstract}

Objective: To examine whether an optimised intervention is a more cost-effective option than TAU for improving agitation and quality of life in nursing home residents with clinically significant agitation and dementia.

Design: A cost effectiveness analysis within a cluster-randomised factorial study in 69 care homes with 549 care home residents was conducted. Each cluster was randomized to receive either the WHELD intervention or TAU for nine months. Health and social care costs, agitation and quality of life outcomes were evaluated.

Results: Improvements in agitation and quality of life were evident in residents allocated to the WHELD intervention group. The additional cost of the WHELD intervention was offset by the higher health and social care costs incurred by TAU group residents (mean difference $f 2103 ; 95 \% \mathrm{Cl}-13$ to $4219)$.

Conclusion: The WHELD intervention has clinical and economic benefits when used in residents with clinically significant agitation.

Keywords: Alzheimer, Dementia, Costs, Care home, Nursing home, Agitation, Fees. 


\section{Background}

The vast majority of care home residents have dementia or severe memory problems and the cost of supporting them has been estimated to be between $f 7$ and $f 13$ billion annually, depending on the proportion of self-funders. ${ }^{1}$ For all residents, care home fees account for the majority (95\%) of the costs. Of the services provided by agencies outside the care home, hospital services are the most expensive category. Hospital costs contribute $3 \%$ towards the total costs and primary and secondary care costs contribute $2 \%$. When care home fees are excluded, almost $50 \%$ of the costs are accounted for by hospital costs. ${ }^{1}$ These high costs are not only attributable to having dementia but are also associated with comorbidities which can co-occur.

Neuropsychiatric symptoms are known to occur over the course of dementia. Behavioural symptoms such as agitation, anxiety and aggression, are among some of the most upsetting symptoms for individuals with the disease and can have a substantial impact on quality of life. ${ }^{2}$ Improving behaviour, functioning and quality of life of residents in care homes pose a challenge for care home managers because there are mixed findings from studies evaluating routinely used pharmacological and non-pharmacological therapies.

Anti-dementia medications are widely considered to be effective in people with Alzheimer's disease (AD). Acetylcholinsterase inhibitor (AChEI) (also known as cholinesterase inhibitors) include drugs such as donepezil, rivastigmine, memantine and galantamine and combinations of these drugs are being used. For people with mild to moderate AD neither galantamine nor rivastigmine had any significant effects on behaviour and relative to donepezil or galantamine significantly better effects on behaviour was observed with rivastigmine ${ }^{3}$. When treated with cholinesterase inhibitors modest improvements in functioning and clinical global impression were found in people with moderate to severe $A D^{4,5}$ and in people with severe $A D^{6,7}$. Other research into the use of cholinesterase inhibitors in people with moderate to severe $A D$ investigated if there were benefits from continuing treatment and whether initiating memantine in the course of the disease was beneficial ${ }^{8}$. Howard and colleagues ${ }^{8}$ found that continued treatment with donepezil was associated with modest cognitive and functional benefits, and the start of memantine was associated with significantly better impacts on cognition and function, although the magnitude of the advantage was smaller than it was with donepezil.

Although the evidence on cholinesterase inhibitors differs by severity of dementia, the evidence on the value of using antipsychotic medications is more conclusive when treating psychiatric symptoms. The evidence although riddled with complexity highlights consensus on modest benefits when treating psychiatric symptoms and the significant risks of antipsychotic drug use ${ }^{9-13}$. Person centred 
care has emerged as an alternative way of caring for someone with dementia, and shifts attention from behaviour to what may be causing or contributing to the behaviour ${ }^{14}$. The Caring for Aged Dementia Care Residents (CADRES) study reported a reduction in agitation in nursing home residents through personalized care techniques based on an individuals' preferences and needs. ${ }^{15}$ However, there have been other studies that have explored the direct impacts of personalized interventions on quality of life. ${ }^{16}$

These concerns are important, given scarce resources to determine whether interventions to improve the quality of life of care home residents represents good value for money. A systematic review of the literature on economic evaluations of dementia found a small number of studies that investigate interventions in care home residents, but these tended to focus on a limited number of study participants. ${ }^{17}$ As part of a review of the literature, one study of care home interventions found many manual-based studies but only six were randomized controlled trials (RCTs) investigating quality of life as a measure of outcome but none were cost effectiveness studies. ${ }^{18}$ The Well-being and Health for people with Dementia (WHELD) study sought to examine the effectiveness and cost-effectiveness of a programme of care combining components intended to improve the quality of life of care home residents with dementia and agitation. The components included person-centred care, management of agitation and psychosocial approaches. The effectiveness findings have been published elsewhere. ${ }^{18}$ In this article, we examine the costeffectiveness of an intervention WHELD with treatment as usual (TAU) to improve the wellbeing of people with dementia in care homes. ${ }^{19}$

\section{Methods}

Hypothesis

The WHELD randomised control trial compared an optimised intervention plus TAU compared to TAU for care home residents with dementia and agitation (Cohen Mansfield Agitation Inventory; ${ }^{20}$ CMAI>29). The current analysis focussed on participants with clinically significant levels of agitation (CMAI >40). The economic hypothesis was that the additional cost of WHELD and TAU compared with TAU would be offset by the improvements in agitation and quality of life in addition to any savings in the use of health and social care services.

Study design and participants

WHELD was a two-arm cluster-randomized controlled trial, conducted in 69 care homes, with a minimum cluster size of 12 participants. Each cluster was randomized by dynamic allocation to 
receive either the optimised WHELD intervention or TAU over nine months. There were three recruiting hubs based in South London, North London and Buckinghamshire in the UK.

Participants in the trial met the clinical criteria for dementia as defined by the Clinical Dementia Rating ${ }^{20}$ with a score greater than 1; were at stage 4 or greater on the Functional Assessment Staging; ${ }^{21}$ and for the current analysis met the clinical criteria for significant agitation (a score of greater than 40 , as defined by the CMAI). All residents meeting the eligibility criteria within the homes were invited to participate.

Interventions

The details of the WHELD intervention are described in detail elsewhere ${ }^{18}$. In brief, the WHELD intervention was delivered as a streamlined, manualised version of the intervention focusing on Person Centred Care (PCC) training for staff; promoting tailored social interaction; and a care home based system for triggering appropriate review of antipsychotic medications by General Practitioners (GPs). Care home staff received training from a research therapist. Two lead care staff members ("WHELD Champions", also referred to as "Champions") were nominated in each care home and received support from the therapist over a period of four months (one training day per month). The staff also received so additional coaching, supervision and regular review with the therapist over the nine-month period. The Champions were then responsible for the delivery and dissemination of the intervention in each care home. In addition, GPs associated with each home were sent educational materials about the programme but there were no proactive education sessions delivered by the research team.

The delivery of the WHELD intervention was adapted for the care setting and involved: stand-alone training sessions, modelling skills, building sessions into daily routines, working with the resident to develop activities that are personalized and tailored to meet their needs, care home team formulation, medication review and goal planning sessions.

The care homes in the control group received treatment as usual (TAU) alone. Treatment as usual was provided by service providers external to the care home and included a range of health and social care services such as hospital inpatient, outpatient, day hospital, accident and emergency services, primary care, community health care and social care services.

\section{Outcomes}

The primary measure of outcomes at baseline (before randomisation) and at nine months following randomisation was quality of life measured by the DEMQOL proxy, ${ }^{22,23}$ which assesses the healthrelated quality of life (HRQoL) of people with dementia. A secondary measure of outcome was 
agitation, measured by the Cohen-Mansfield Agitation Inventory (CMAI), which is a caregiver's rating questionnaire to specify agitated behaviour.

Resource use and costs

Economic data for each individual in the study was collected using an adapted version of the Client Service Receipt Inventory (CSRI) which has been used in previous studies of care home residents with dementia. ${ }^{1,24,25}$ It includes questions about the resident's sociodemographic profile, care home charges, and use of health and social care services. It was administered retrospectively at two data collection time points: by interview at baseline covering the previous three-month period and nine months after randomization (covering the previous three months). In addition, the staffing inputs of the WHELD intervention were measured and included time spent by the therapist and champion in training, supervision and preparation.

Total costs for each care home resident were derived by summing three main cost categories: intervention costs, resident fees and costs of health and social care services provided by organisations external to the care homes.

The intervention cost for each home was apportioned across the number of study participants in the care home to derive an intervention cost for each care home resident in the study. To these costs we added the cost for an antipsychotic review for those allocated to the WHELD intervention and TAU and were on antipsychotics during the trial. The Champions were likely to be staff from different professions within the care home. For each of these professions we obtained separate data on the professional's cost per hour and derived an average cost per hour for a Champion and that of a therapist. Data on the time inputs for training, supervision and delivery of the WHELD intervention was combined with the cost per hour for each professional who conducted the following: received the training, were supervised and were involved in the supervision, and those who delivered the intervention. The supervisors were also supervised by three senior principal investigators during the study. These research-related costs were not included.

The resident fees were collected from the care homes. Where data on study resident's fees could not be elicited, we asked the care home for the typical weekly fee for residents with a similar level of need to the participant in the study. Where both of these data were missing, the resident's fees were imputed based on the average fee for study residents in that care home. Weekly fees were extrapolated to a nine-monthly figure.

Total health and social care costs consisted of services provided by agencies external to the care home: hospital inpatient, outpatient, day hospital, accident and emergency services, primary care, community health care and ambulatory care. Services and support costs provided by external 
agencies were calculated by multiplying the frequency and intensity of resources collected from the CSRI by appropriate unit costs. Unit costs (2014/2015 price level) were obtained from widely used published sources (Table 1$)^{26,27 .}$

Cost-effectiveness analysis

The primary economic evaluation at nine months (i.e. a cost-effectiveness analysis) included cost and outcomes for the two randomisation groups - the WHELD intervention and TAU - from a health and social care perspective. The cost effectiveness analysis focused on two outcomes: CMAl and DEMQOL proxy. Societal weights were applied to DEMQOL proxy scores ${ }^{28}$ to calculate utility values. Quality-adjusted life years (QALYs) were calculated by 'area-under-the-curve' analysis, with linear interpolation between baseline and nine-months follow up assessments points.

Statistical analyses

Analyses were conducted on residents allocated to the care homes receiving the WHELD intervention and TAU. Residents were analysed in groups to which they were allocated irrespective of drop out.

We imputed CMAI scores at follow up for participants with clinically significant agitation who did not have follow up CMAI data, in the following way. Figure 1 The CONSORT diagram (Figure 1) shows the people with and without clinically significant agitation who were assessed at follow-up and on whom a complete case clinical analysis was conducted. Of those 257 (WHELD + TAU) and 296 (TAU); 200 (WHELD + TAU) and 181 (TAU) participants had clinically significant agitation. We imputed CMAI scores for those who did not have follow up CMAI data and needed to have their scores imputed: 67 (25.1\%) (WHELD + TAU) and 101 (35.8\%) (TAU).

The missing service use data (either contacts or duration of contact) and outcomes were imputed to facilitate the estimation of subtotal and total costs. Ordinary Least Squares (OLS) regression-based single imputation - with a variety of baseline covariates including personal characteristics, value of variable being imputed and care home indicator - was used to impute missing values at follow-up assessment

For the base case model we selected individuals in the study with a CMAI score $>40$ and ran multilevel mixed effect models. In the cost and outcome models, we controlled for baseline values of cost, outcomes, site and age. The adjusted total health and social care cost and outcomes models also included the treatment variable as a random effect at the care home level. We accounted for clustering by allowing the model intercept and treatment variable coefficient (i.e. treatment effect) to vary by care home. 
The cost-effectiveness of the WHELD intervention and TAU compared to TAU alone was evaluated by calculating incremental cost-effectiveness ratios (ICERs). The ICER is defined as the between group difference in mean costs divided by the between group difference in mean outcome. Incremental cost-effectiveness ratios were calculated in turn for each measure of outcome: QALY and CMAI. Using these ratios, we were able to determine a cost per unit improvement in QALY and the cost per point improvement in agitation (CMAl scale).

Bootstrapping was conducted with the mixed effects models to generate confidence intervals that can be used to capture parameter uncertainty around the estimates in the cost-effectiveness analysis. The 1000 treatment effect replications from this bootstrapping process were plotted on cost-effectiveness planes and used to construct cost-effectiveness acceptability curves (CEAC), which are also standard practice in trial based economic evaluations. ${ }^{29}$

Cost-effectiveness acceptability curves

The CEAC is an established construct for presenting parameter uncertainty. The willingness-to-pay threshold ICERs are shown on the horizontal axis and give hypothetical values of societal willingnessto-pay for each additional unit increase in outcome per average patient. ${ }^{30,31}$ The vertical axis gives the probability that the WHELD intervention and TAU is relatively cost-effectiveness relative to TAU alone at each of these threshold ICERs. Using the net benefit approach, monetary values of incremental effects and incremental costs were combined, and net benefit (NB) derived as: NB $=\lambda x$ $\left(\right.$ effect $_{b}-$ effect $\left._{a}\right)-\left(\operatorname{cost}_{b}-\operatorname{cost}_{a}\right) ; \lambda$ is willingness-to-pay for a unit improvement in effectiveness (CMAI, QALYs), and ' $a$ ' and ' $b$ ' denote TAU and WHELD interventions, respectively.

The data analyses were conducted using SPSS for Windows release 12.0.1 (SPSS Inc., Chicago, IL, USA; 1989-2001) and STATA 14 for Windows (StataCorp, College Station, TX, USA; 1985 - 2004). Sensitivity analyses

To explore the sensitivity of the results to alternate statistical methods the analyses were repeated using an OLS model with cluster adjusted standard errors. This included the same control variables as those in the base-case model.

\section{Results}

Participants

971 residents had their care homes randomised as shown in the Consolidated Statement of Reporting Trials (CONSORT) (Figure 1). Of the 971 residents, 549 residents ( $57 \%$ of residents recruited to the trial) met the clinical criteria for agitation at baseline. Of those residents with a clinically significant agitation, there were 282 residents in the TAU group and 267 in the WHELD 
intervention and TAU group. The baseline characteristics were broadly comparable across the intervention arms (Table 2).

For those with clinically significant agitation, there was a high response rate for service use data collected at both assessment time points. At baseline, in the TAU group service use data was collected on 281 (99.6\%) care home residents. For one (0.4\%) individual in this group no service use data was collected and in the intervention group service use data was collected on 267 (100\%) residents. At nine months, the number of care home residents with any service use data in the TAU group fell to $231(82 \%)$ and in the intervention group there were $199(75 \%)$ individuals.

Costs and cost effectiveness

The additional cost of delivering the intervention compared to TAU was $f 2,629$ (SD 516). The majority $(53 \%$ or $f 1,367)$ of these costs related to the time the Champion spent in training and receiving feedback from the supervising therapists. The therapist time spent preparing for the training, actual training and supervision of the Champion contributed the remaining $47 \%(£ 1,262)$ of the cost.

There were notable changes in the pattern of health and social care cost over the time of the study. The health and social care costs for residents in the TAU group were 1.2 times higher ( $£ 34215$ ) than those in the WHELD intervention and TAU group ( $₫ 29483$ ) in the nine-month period following intervention. However, the unadjusted difference in total health and social care costs including the intervention did not achieve statistical significance ( $f 2103 ; \mathrm{Cl}-13$ to 4 219). The WHELD intervention conferred a significant improvement in agitation and quality of life compared to treatment as usual. The unadjusted difference in CMAI score was 5.16 (95\% Cl 1.11 to $9.20, p=0.01$ ) and for DEMQoLproxy score, $3.23(95 \% \mathrm{Cl}-5.54$ to $-0.93, \mathrm{p}=0.01)$ (Table 3$)$.

In the base case analysis we compared the intervention using multilevel mixed models adjusting for a range of covariates and found that the additional costs incurred by the WHELD intervention did not result in significantly higher overall costs to health and social care relative to TAU alone, but provided QALY gains and improvements in agitation (Table 4). As compared with TAU, the WHELD intervention was no more costly (95\% confidence interval [CI], -2578 to 179) per person and provided an additional 0.01 QALYs per person $(95 \% \mathrm{Cl}, 0.001$ to 0.018$)$ and 3.72 points of improvement in agitation per person $(95 \% \mathrm{Cl}, 1.15$ to 6.38); the corresponding ICERs were - $f 137978$ per QALY gained $(95 \% \mathrm{Cl},-733656$ to 34483$)$ and $-£ 348$ per point improvement in agitation $(95 \% \mathrm{Cl}$, -1156 to 79$)$.

The CEACs showed that the probabilities of cost-effectiveness across a wide range of a decision maker's willingness to pay for a unit improvement in outcome. These were similar for both outcome 
measures. Comparing costs and both measures of outcome in turn, the WHELD intervention and TAU was consistently the dominant intervention (Figures 2-3). The CEAC for QALYs started at 95\% probability that WHELD and TAU is relatively cost-effective compared to TAU alone and this gradually rose to $100 \%$ at a willingness-to-pay threshold ICER of $£ 70000$ for each QALY gained (Figure 4). If decision makers were willing to pay $£ 200$ for each point improvement in agitation the probability that the WHELD intervention and TAU is cost effective is as high as $100 \%$ (Figure 5).

Sensitivity analysis

The OLS models with cluster robust standard errors produced broadly similar results to the multilevel models for clinical outcomes. QALY and CMAI models gave similar results to the base case (QALY: coefficient $0.009 ; p=0.01 ; 95 \% \mathrm{Cl}-0.000$ to $0.021 ; \mathrm{CMAl}$ : coefficient $-3.49 ; \mathrm{p}=0.04 ; 95 \% \mathrm{Cl}$ 0.33 to 6.985$)$. The WHELD intervention and TAU was no more costly than TAU ( $p=0.51 ; 95 \% \mathrm{Cl}-2$ 680 to 1094$)$.

\section{Discussion}

This is the first cost effectiveness study to report on the value to nursing home residents of using interventions which consider the reduction of antipsychotic use. A recently published systematic review highlighted the lack of clinical and economic reporting of antipsychotic use in nursing homes. $^{32}$

In the WHELD factorised controlled trial of 549 care home residents with dementia and clinically significant agitation (CMAI>40), we found that the WHELD intervention when added to treatment as usual was more cost effective than treatment as usual alone, when considering health and social care costs and improvements in QALYs and agitation.

Costs and cost effectiveness

The evaluation of costs found that the additional intervention costs resulted in notable changes in the pattern of health care over the time of the study. There were higher health and social care costs in the TAU group compared to those in the WHELD intervention group suggesting that the additional costs of the intervention resulted in health and social care cost savings when direct action is taken to improve the quality of life and agitation of residents with dementia living in care homes. Both agitation and QALYs improved significantly over the intervention period. The assessment of cost effectiveness and parameter uncertainty confirmed that the WHELD intervention and TAU would have a higher probability of being cost effective compared to TAU alone for a wide range of societal willingness-to-pay thresholds. The results were broadly similar when using multilevel modelling and OLS models. The largest difference between the methods was shown in the results for costs: the 
difference in costs was around $£ 400$ less in the OLS model with the bootstrapped confidence interval crossing 0 to the positive part of the number line. There was more uncertainty when using the OLS model approach around the conclusion that the WHELD intervention was cheaper. When the impact of the WHELD intervention on agitation and quality of life were considered, the WHELD intervention was more effective than TAU. These results are mainly relevant to residents with clinically significant agitation in dementia and may be less so for residents for whom agitation is less severe.

\section{Strengths and limitations}

We adopted a health and social care perspective which can be used to inform reimbursement decisions, particularly in sectors with overstretched budgets. A health and social care viewpoint is also in line with the approach adopted by decision makers in Europe, Australia and Canada. ${ }^{33}$ Despite the widespread use of such a narrow point of view which considers only the cost and outcome impacts to health and social care, there is some recognition that decisions that are made using evidence that omit the impacts to other stakeholders such as caregivers for example, could potentially result in welfare losses to these stakeholders.

Consistent with a health and social care viewpoint, we did not include any costs to unpaid carers of the care home residents. We know from published research that the time spent by relatives and friends providing unpaid support to care home residents is not insubstantial; with one estimate suggesting that unpaid caregivers spend an average of 36 hours per month providing support to individuals in long term care settings ${ }^{34}$. However, we also recognise that there are theoretical challenges - noted in detail elsewhere ${ }^{35,36}$-to including the costs and effects that fall more widely on society.

In the economic evaluation the DEMQoL-proxy was used as the measure of outcome on which to derive QALYS. We were unable to use the study participant's report of their own quality of life assessed using the DEMQoL to derive QALYs, as there was a high degree of missing data (83\% missing entries), whereas the proxy data had only a third of entries missing. The cost effectiveness finding was therefore dependent on who was reporting quality of life as there are important differences between self-report and carer-proxy reports which suggest that these questionnaires cannot necessarily be used interchangeably. ${ }^{37}$ Therefore, these results should be interpreted with caution.

It is important to note that this economic evaluation covers a nine-month follow-up period and although this is a relatively long time in the context of trial-based evaluations; additional research would be required to investigate even longer term trends and increments. The authors are not 
aware of any model-based evaluations of optimised interventions such as WHELD in nursing home residents with dementia.

\section{Conclusions}

The WHELD intervention trial offers new evidence on costs and outcomes in the use of an optimised intervention which focused on changing the culture in care homes through the use of a Champion. The trial suggests a strong case for the introduction of the WHELD intervention - not only on clinical grounds but also on economic grounds - for residents with clinically significant agitation. This is of relevance for commissioning bodies given the findings on cost effectiveness. For example, a report jointly produced by the British Geriatrics Society and the Care Quality Commission, revealed a patchy and chaotic approach to commissioning and providing health care services for care home residents. There were missing services and care gaps identified by the report which included medication review and care planning. This study has shown that proactive medical review and more responsive support for care home residents can reduce inpatient admissions and emergency hospital admissions, while improving the outcomes of residents. However, further research is needed to build a better understanding of how these interventions impact the quality of life of the care home resident living with severe agitation and dementia.

\section{Acknowledgements}

We are very grateful to the participating care homes. We would like to thank the members of the Steering Committee: Professor Robin Jacoby (Chair), Professor Clive Ballard, Professor Dawn Brooker, Des Kelly, Dr Lindsey Roberts, Geir Selbaek, Graham Stokes. The WHELD research team acknowledges the support of the National Institute for Health Research, through the Dementia and Neurodegenerative Research Network, DeNDRoN. We would like to thank the NIHR Biomedical Research Unit for Dementia at King's College London/Maudsley hospital for supporting the involvement of Clive Ballard and the Biomedical Research Centre for mental Health at King's College London/Maudsley hospital for supporting the care home research network which supported participating care homes.

\section{Financial Disclosure}

The article presents independent research funded by the National Institute for Health Research (NIHR) under its Programme Grants for Applied Research (PGFAR) Programme grant reference number: RPPG-0608-10133. The views expressed are those of the authors and are not necessarily those of the NHS, NIHR or the Department of Health. 
Table 1. Unit costs (2014/2015 prices)

\begin{tabular}{|l|c|}
\hline Service & Unit cost \\
& $\mathbf{2 0 1 4 / 2 0 1 5}$ \\
\hline Hospital visit; per night ${ }^{23}$ & 608 \\
\hline Accident and emergency; per visit ${ }^{23}$ & 44 \\
\hline Day hospital; per attendance ${ }^{22}$ & 704 \\
\hline Outpatient attendance; per attendance ${ }^{\mathbf{2 2}}$ & 112 \\
\hline Memory clinic; per hour ${ }^{\mathbf{2 2}}$ & 424 \\
\hline Paramedic; cost per attendance ${ }^{\mathbf{2 2}}$ & 99 \\
\hline District nurse; per minute ${ }^{\mathbf{2 2}}$ & 0.73 \\
\hline Practice nurse; per minute ${ }^{\mathbf{2 2}}$ & 0.72 \\
\hline Specialist nurse; per minute ${ }^{\mathbf{2 2}}$ & 0.87 \\
\hline Physiotherapist; per minute ${ }^{\mathbf{2 2}}$ & 0.57 \\
\hline GP; per minute ${ }^{\mathbf{2 2}}$ & 3.20 \\
\hline
\end{tabular}


Table 2. Baseline resident characteristics by intervention arm

\begin{tabular}{|c|c|c|}
\hline & $\begin{array}{l}\text { WHELD intervention } \\
\text { and TAU }\end{array}$ & TAU alone \\
\hline Age in years, mean (sd) & $\begin{array}{l}n=267 \\
85(8.9)\end{array}$ & $\begin{array}{c}n=282 \\
84(9.0)\end{array}$ \\
\hline Gender (male); n (\%) & $\begin{array}{l}\mathrm{n}=267 \\
91(34)\end{array}$ & $\begin{array}{l}\mathrm{n}=282 \\
82(29)\end{array}$ \\
\hline Ethnicity (white) n (\%) & $\begin{array}{l}n=267 \\
253(95)\end{array}$ & $\begin{array}{c}n=282 \\
271(96)\end{array}$ \\
\hline $\begin{array}{l}\text { Marital status (married/long-term partnership) } \\
\mathrm{n}(\%)\end{array}$ & $\begin{array}{l}n=263 \\
59(22)\end{array}$ & $\begin{array}{l}n=279 \\
67(24)\end{array}$ \\
\hline Next of kin: yes; $n(\%)$ & $\begin{array}{l}n=267 \\
254(95)\end{array}$ & $\begin{array}{c}n=282 \\
267(95)\end{array}$ \\
\hline CMAl score, mean (sd) & $\begin{array}{l}n=267 \\
59(18)\end{array}$ & $\begin{array}{l}n=282 \\
61(19)\end{array}$ \\
\hline CSDD score, mean (sd) & $\begin{array}{l}n=264 \\
8(5)\end{array}$ & $\begin{aligned} n & =279 \\
8 & (5)\end{aligned}$ \\
\hline CDR score, mean (sd) & $\begin{array}{l}n=267 \\
14(3)\end{array}$ & $\begin{array}{l}n=282 \\
14(4)\end{array}$ \\
\hline $\mathrm{NPI}$ - score, mean (sd) & $\begin{array}{l}n=264 \\
21(16)\end{array}$ & $\begin{array}{l}n=279 \\
21(16)\end{array}$ \\
\hline DEMQoL proxy, mean (sd) & $\begin{array}{l}n=264 \\
99(13)\end{array}$ & $\begin{array}{l}\mathrm{n}=281 \\
98(12)\end{array}$ \\
\hline DEMQoL score, mean (sd) & $\begin{array}{c}n=84 \\
85(18)\end{array}$ & $\begin{array}{c}n=79 \\
86(16)\end{array}$ \\
\hline Total health and social care costs, $\mathrm{f} ;$ mean (sd) & $\begin{array}{c}n=266 \\
9,979(2579)\end{array}$ & $\begin{array}{c}n=282 \\
11,013(4627)\end{array}$ \\
\hline
\end{tabular}


Table 3 Unadjusted mean costs and mean cost differences at baseline and over 9 months $(f, 2014-2015)$

\begin{tabular}{|c|c|c|c|c|c|c|}
\hline \multirow[b]{2}{*}{ Cost categories } & \multicolumn{2}{|c|}{$\begin{array}{l}\text { WHELD intervention } \\
\text { and TAU }\end{array}$} & \multicolumn{2}{|c|}{ TAU alone } & \multicolumn{2}{|c|}{$\begin{array}{l}\text { WHELD Intervention and TAU } \\
\text { vs TAU alone }\end{array}$} \\
\hline & Mean $(f)$ & $\begin{array}{l}\text { SD } \\
(\mathrm{f})\end{array}$ & Mean $(\mathrm{f})$ & $\begin{array}{l}\text { SD } \\
(f)\end{array}$ & $\begin{array}{r}\text { Unadjusted } \\
\text { mean difference } \\
(\mathrm{f})\end{array}$ & $95 \% \mathrm{Cl}$ \\
\hline \multicolumn{7}{|l|}{ Baseline -3 months ( $n=549)$} \\
\hline Total health and social care costs & 9980 & $(2579)$ & 11013 & $(4627)$ & -1033 & $(409 \text { to } 1658)^{\wedge}$ \\
\hline WHELD intervention & 2629 & 516 & - & - & 2629 & (-2 701 to -2557$)$ \\
\hline \multicolumn{7}{|l|}{ Follow up - 9-months $(n=430)$} \\
\hline Accommodation fee & 28382 & $(10851)$ & 32723 & $(11142)$ & -4341 & $(2252 \text { to } 4630)^{\wedge}$ \\
\hline Hospital & 273 & $(1238)$ & 325 & $(1582)$ & -52 & $(-216$ to 320$)$ \\
\hline Primary care & 701 & $(300)$ & 1007 & $(270)$ & -306 & $(-251$ to 360$)$ \\
\hline Community health & 81 & (297) & 72 & (229) & 9 & $(-60$ to 42$)$ \\
\hline Emergency & 46 & (110) & 88 & $(222)$ & -42 & ( 10 to -75$)$ \\
\hline $\begin{array}{l}\text { Total health and social care costs incl. } \\
\text { intervention costs }\end{array}$ & 32112 & $(10961)$ & 34215 & $(11326)$ & -2103 & (-13 to 4219$)$ \\
\hline
\end{tabular}


Table 4 Mean adjusted incremental cost and effect, and cost-effectiveness ratios ( $f, 2014 / 2015)$ over 9 months

\begin{tabular}{|c|c|}
\hline & $\begin{array}{l}\text { WHELD Intervention and TAU vs } \\
\text { TAU alone }\end{array}$ \\
\hline \multicolumn{2}{|l|}{ Mean incremental cost* $(£, 2014 / 15$ prices $)$ mean $(95 \% \mathrm{Cl})$} \\
\hline Health and social care & $-1294(-2577$ to 179$)$ \\
\hline \multicolumn{2}{|l|}{ Mean Incremental effect $(95 \% \mathrm{Cl})^{*}$} \\
\hline $\begin{array}{l}\text { CMAl score (reversed so higher scores mean better } \\
\text { outcomes) }\end{array}$ & $3.72(-1.15$ to 6.38$)$ \\
\hline DEMQoL - Proxy score (higher scores better outcomes) & $1.96(0.38$ to 3.24$)$ \\
\hline \multicolumn{2}{|l|}{ Incremental cost-effectiveness ( $€$, 2014/2015 prices)* } \\
\hline Health and social care cost/CMAI & $-348(-1709$ to -107$)$ \\
\hline Health and social care cost/ QALY & $-137978(-733656$ to 34483$)$ \\
\hline
\end{tabular}

*Adjusted for site, age, gender, CMAl score before and randomisation and total costs at baseline. 
Figure 1. CONSORT diagram showing flow of participants through the study. TAU arm - treatment as Usual. WHELD arm - WHELD plus TAU. Abbreviations: WHELD, Well-being and Health for People with Dementia; TAU, treatment as usual

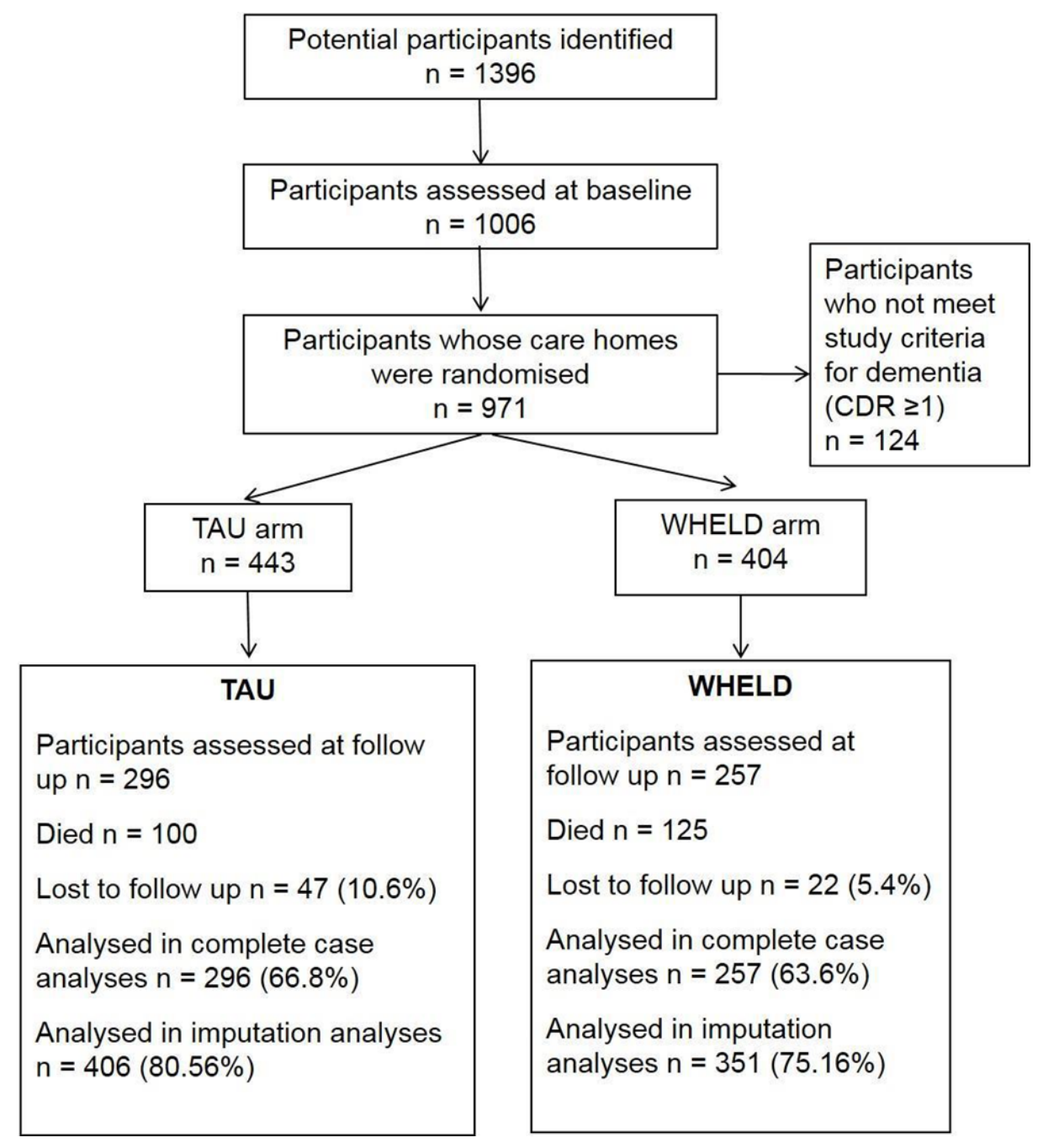


Figure 2. Scatter plot: WHELD intervention and TAU vs. TAU alone; health and social care perspective, with effectiveness measured in QALYs

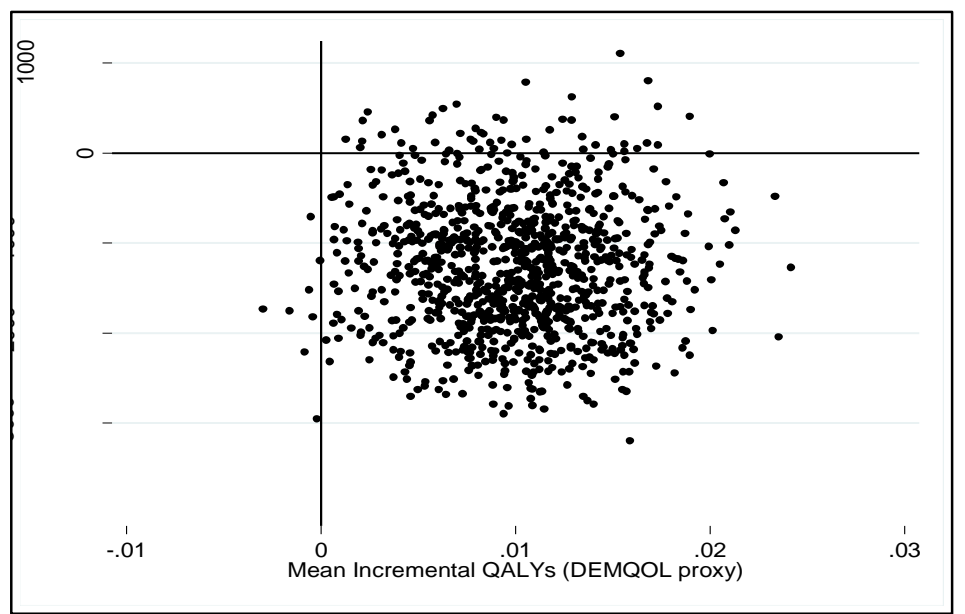

Figure 3. Scatter plot: WHELD intervention and TAU vs. TAU alone; health and social care perspective, with effectiveness measured in CMAI

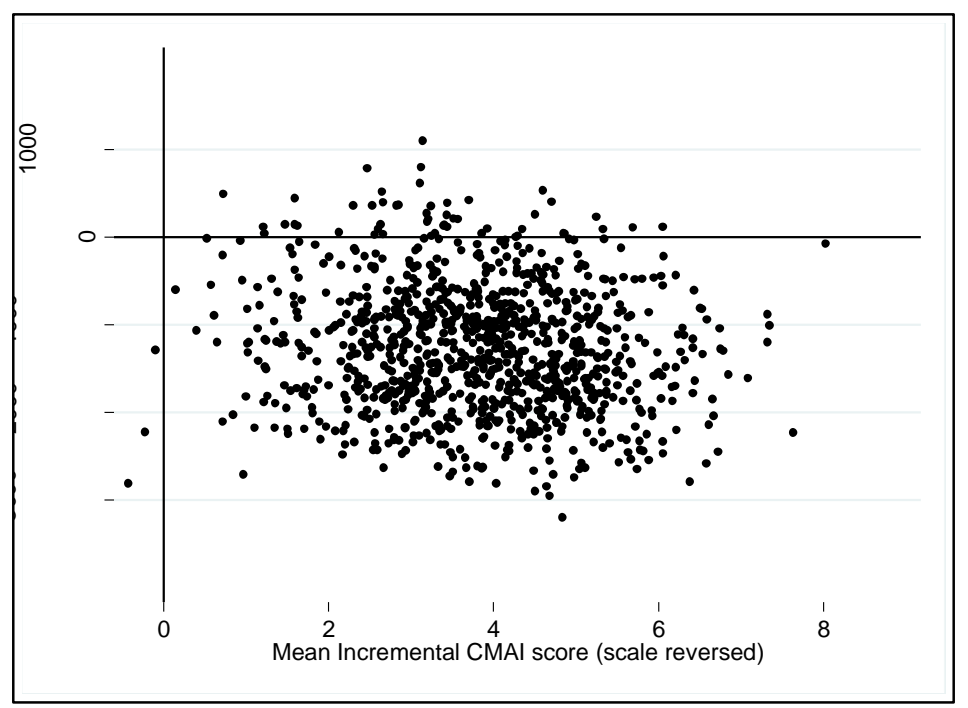

Figure 4. Cost-effectiveness acceptability curve: WHELD intervention and TAU vs. TAU alone; health and social care perspective, with effectiveness measured in QALYs

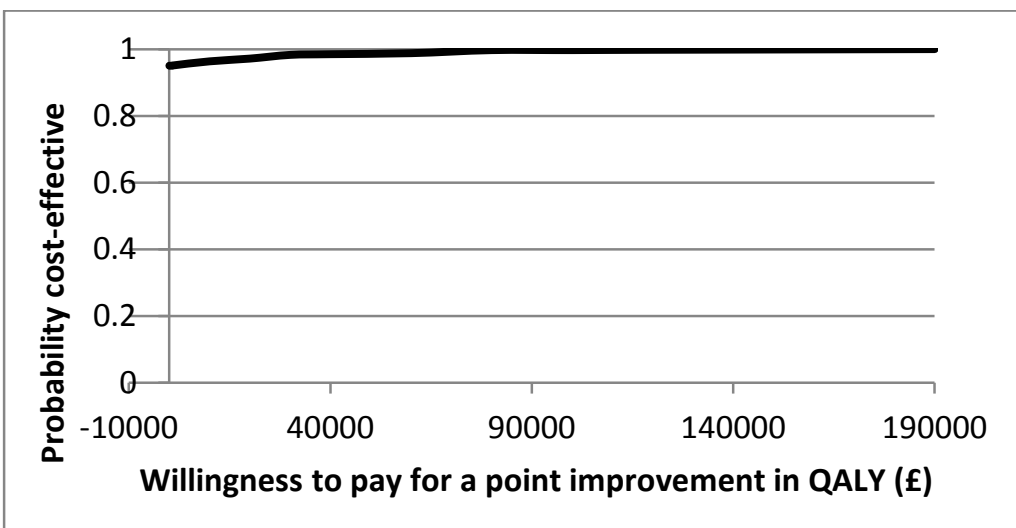


Figure 5. Cost-effectiveness acceptability curve: WHELD intervention and TAU vs. TAU alone; health and social care perspective, with effectiveness measured in CMAI

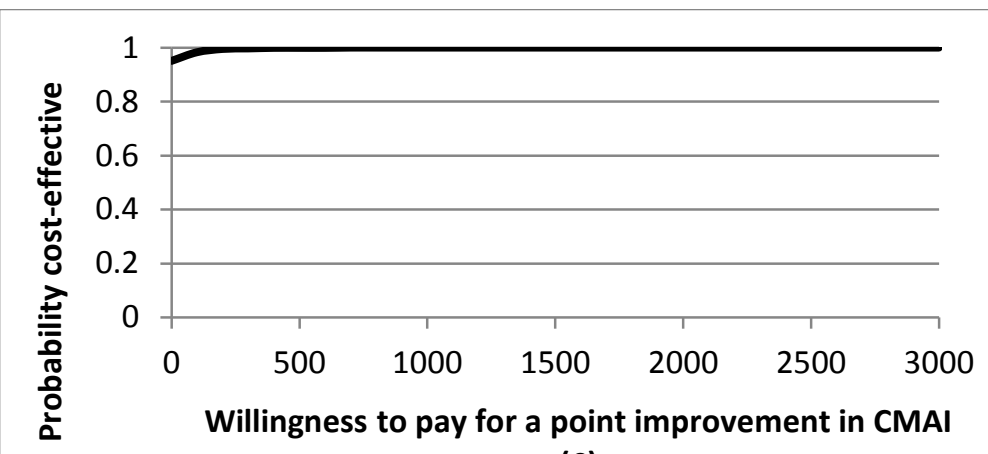

(f) 


\section{References}

1. Romeo R, Knapp M, Salverda S, Orrell M, Fossey J, Ballard C. The cost of care homes for people with dementia in England: a modelling approach. International Journal of Geriatric Psychiatry. 2017 Dec 1;32(12):1466-75.

2. Wetzels RB, Zuidema SU, de Jonghe JF, Verhey FR, Koopmans RT. Determinants of quality of life in nursing home residents with dementia. Dementia and Geriatric Cognitive Disorders. 2010;29(3):18997.

3. National Institute for Health and Care Ecxcellence (2016) Dementia: supporting people with dementia and their carers in health and social care. NICE Guidelines CG42:

https://www.nice.org.uk/guidance/cg42.

4. Feldman H, Gauthier S, Hecker J et al. (2001) A 24-week, randomized double blind placebocontrolled study of donepezil in moderate to severe Alzheimer's disease. Neurology 57 (4): 613-20.

5. Tariot PN, Cummings JL, Katz IR et al. (2001) A randomized double blind placebo controlled study of the efficacy and safety of donepezl in patient with Alzheimer's disease in the nursing home setting. Journal of the American Geriatric Society 49: 1590-9.

6. Feldman H, Gauthier S, Hecker J et al. (2005). Efficacy and safety of donepezil in patients with severe Alzheimer's disease: a subgroup analysis from a randomised placebo-controlled trial. International Journal of Geriatric Psychiatry 20: 559-69.

7. Winblad B, Kilander L, Eriksson S et al. (2006) Donepezil in patients with severe Alzheimer's disease: double-blind, parallel-goup, placebo-controlled study. Lancet 367: 1057-65.

8. Howard RJ, Juszczak E, Ballard CG, Bentham P, Brown RG, Bullock R, Burns AS, Holmes C, Jacoby R, Johnson T, Knapp M. Donepezil for the treatment of agitation in Alzheimer's disease. New England Journal of Medicine. 2007 Oct 4;357(14):1382-92.

9. Ballard C, Howard R. Neuroleptic drugs in dementia: benefits and harm. Nature Reviews Neuroscience. 2006 Jun;7(6):492.

10. Banerjee S (2008) The Use of Antipsychotic Medication for People with Dementia: Time for Action. London: Department of Health.

11. Schneider LS, et al. "Effectiveness of Atypical Antipsychotic Drugs in Patients with Alzheimer's Disease". The New England Journal of Medicine. 2006. 355(15):1525-38.

12. Ballard C, Waite J. The effectiveness of atypical antipsychotics for the treatment of aggression and psychosis in Alzheimer's disease. Cochrane Database Syst Rev. 2006 Jan 25;(1):CD003476.

13. Ballard C, et al. A randomised, blinded, placebo-controlled trial in dementia patients continuing or stopping neuroleptics (the DART-AD trial). PLoS Med. 2008 Apr 1;5(4):e76.

14. Fossey J, Masson S, Stafford J, Lawrence V, Corbett A, Ballard C. The disconnect between evidence and practice: a systematic review of person-centred interventions and training manuals for care home staff working with people with dementia. International Journal of Geriatriatric Psychiatry. 2014;29(8):797-807. pmid:24535885 
15. Chenoweth L, King MT, Jeon YH, Brodaty H, Stein-Parbury J, Norman R, Haas M, Luscombe G. Caring for Aged Dementia Care Resident Study (CADRES) of person-centred care, dementia-care mapping, and usual care in dementia: a cluster-randomised trial. The Lancet Neurology. 2009 Apr 1;8(4):317-25.

16. Livingston G, Kelly L, Lewis-Holmes E, Baio G, Morris S, Patel N, Omar RZ, Katona C, Cooper C. Non-pharmacological interventions for agitation in dementia: systematic review of randomised controlled trials. The British Journal of Psychiatry. 2014 Dec 1;205(6):436-42.

17. Knapp M, lemmi V, Romeo R. Dementia care costs and outcomes: a systematic review. International Journal of Geriatric Psychiatry. 2013 Jun 1;28(6):551-61.

18. Ballard C, Corbett A, Orrell M, Williams G, Moniz-Cook E, Romeo R, et al. (2018) Impact of person-centred care training and person-centred activities on quality of life, agitation, and antipsychotic use in people with dementia living in nursing homes: A cluster-randomised controlled trial. PLoS Med 15(2): e1002500. https://doi.org/10.1371/journal.pmed.1002500.

19. Whitaker R, Fossey J, Ballard C, Orrell M, Moniz-Cook E, Woods RT, Murray J, Stafford J, Knapp M, Romeo R, Carlton BW. Improving Well-being and Health for People with Dementia (WHELD): study protocol for a randomised controlled trial. Trials. 2014 Dec;15(1):284.

20. Cohen-Mansfield J, Marx MS, Rosenthal AS. A description of agitation in a nursing home. Journal of Gerontology. 1989 May 1;44(3):M77-84.

21 Reisberg B. Functional assessment staging (FAST). Psychopharmacology Bulletin. 1988;24(4):6539.

22. Mulhern B, Rowen D, Brazier J, Smith S, Romeo R, Tait R, Watchurst C, Chua KC, Loftus V, Young $T$, Lamping D. Development of DEMQOL-U and DEMQOL-PROXY-U: generation of preference-based indices from DEMQOL and DEMQOL-PROXY for use in economic evaluation. Health technology assessment (Winchester, England). 2013;17(5):1-140.

23. Smith SC, Lamping DL, Banerjee S, Harwood RH, Foley B, Smith P, Cook JC, Murray J, Prince M, Levin E, Mann A. Development of a new measure of health-related quality of life for people with dementia: DEMQOL. Psychological medicine. 2007 May;37(5):737-46.

24. Knapp M, King D, Romeo R, Schehl B, Barber J, Griffin M, Rapaport P, Livingston D, Mummery C, Walker Z, Hoe J. Cost effectiveness of a manual based coping strategy programme in promoting the mental health of family carers of people with dementia (the START (STrAtegies for RelaTives) study): a pragmatic randomised controlled trial. BMJ. 2013 Oct 25;347:f6342.

25. Romeo R, Knapp M, Hellier J, Dewey M, Ballard C, Baldwin R, Bentham P, Burns A, Fox C, Holmes $C$, Katona C. Cost-effectiveness analyses for mirtazapine and sertraline in dementia: randomised controlled trial. The British Journal of Psychiatry. 2012 Dec 14.

26. Curtis L, Burns A. Unit Costs of Health and Social Care 2015. Personal Social Services Research Unit. 2015.

27. Department of Health. 2015. National Health Service Schedule of Reference Costs. 
28. Rowen D, Mulhern B, Banerjee S, van Hout B, Young TA, Knapp M, Smith SC, Lamping DL, Brazier $J E$. Estimating preference-based single index measures for dementia using DEMQOL and DEMQOLProxy. Value in Health. 2012 Mar 1;15(2):346-56.

29. Van Hout BA, Al MJ, Gordon GS, Rutten FF. Costs, effects and C/E-ratios alongside a clinical trial. Health Economics. 1994 Sep 1;3(5):309-19.

30. Claxton K. The irrelevance of inference: a decision-making approach to the stochastic evaluation of health care technologies. Journal of Health Economics. 1999 Jun 30;18(3):341-64.

31. Fenwick E, Byford S. A guide to cost-effectiveness acceptability curves. The British Journal of Psychiatry. 2005 Aug 1;187(2):106-8.

32. Hoyle DJ, Bindoff IK, Clinnick LM, Peterson GM, Westbury JL. Clinical and Economic Outcomes of Interventions to Reduce Antipsychotic and Benzodiazepine Use Within Nursing Homes: A Systematic Review. Drugs \& Aging. 2018 Jan 27:1-2.

33. Sanders GD, Neumann PJ, Basu A, Brock DW, Feeny D, Krahn M, Kuntz KM, Meltzer DO, Owens DK, Prosser LA, Salomon JA. Recommendations for conduct, methodological practices, and reporting of cost-effectiveness analyses: second panel on cost-effectiveness in health and medicine. JAMA. 2016 Sep 13;316(10):1093-103.

34. Max W, Webber P, Fox P. Alzheimer's disease: The unpaid burden of caring. Journal of Aging and Health. 1995 May;7(2):179-99.

35. Brouwer WB, Culyer AJ, van Exel NJ, Rutten FF. Welfarism vs. extra-welfarism. Journal of Health Economics. 2008 Mar 1;27(2):325-38.

36. Drummond MF, Sculpher MJ, Claxton K, Stoddart GL, Torrance GW. Methods for the economic evaluation of health care programmes. Oxford University Press; 2015 Sep 24.

37. Smith SC, Lamping DL, Banerjee S, Harwood R, Foley B, Smith P, Cook JC, Murray J, Prince M, Levin E, Mann A. Measurement of health-related quality of life for people with dementia: development of a new instrument (DEMQOL) and an evaluation of current methodology. Health Technology Assessment (Winchester, England). 2005;9(10):1-93. 